\title{
Magnon-photon coupling in the noncollinear magnetic insulator $\mathrm{Cu}_{2} \mathrm{OSeO}_{3}$
}

\author{
L. V. Abdurakhimov,,${ }^{1, *}$ S. Khan, ${ }^{1}$ N. A. Panjwani, ${ }^{1, \dagger}$ J. D. Breeze,${ }^{2,3}$ M. Mochizuki,${ }^{4}$ S. Seki,${ }^{5}$ Y. Tokura,${ }^{5,6}$ \\ J. J. L. Morton, ${ }^{1}$ and H. Kurebayashi ${ }^{1, \$}$ \\ ${ }^{1}$ London Centre for Nanotechnology, University College London, London WC1H OAH, United Kingdom \\ ${ }^{2}$ Department of Materials, Imperial College London, Exhibition Road, London SW7 2AZ, United Kingdom \\ ${ }^{3}$ London Centre for Nanotechnology, Imperial College London, Exhibition Road, London SW7 2AZ, United Kingdom \\ ${ }^{4}$ Department of Applied Physics, Waseda University, Okubo, Shinjuku-ku, Tokyo 169-8555, Japan \\ ${ }^{5}$ RIKEN Center for Emergent Matter Science (CEMS), Wako 351-0198, Japan \\ ${ }^{6}$ Department of Applied Physics and Quantum Phase Electronics Center (QPEC), University of Tokyo, Tokyo 113-8656, Japan
}

(Received 20 February 2018; revised manuscript received 15 June 2018; published 3 April 2019)

\begin{abstract}
Anticrossing behavior between magnons in the noncollinear chiral magnet $\mathrm{Cu}_{2} \mathrm{OSeO}_{3}$ and a two-mode $X$-band microwave resonator was studied in the temperature range 5-100 K. In the field-induced ferrimagnetic phase, we observed a strong-coupling regime between magnons and two microwave cavity modes with a cooperativity reaching 3600. In the conical phase, cavity modes are dispersively coupled to a fundamental helimagnon mode, and we demonstrate that the magnetic phase diagram of $\mathrm{Cu}_{2} \mathrm{OSeO}_{3}$ can be reconstructed from the measurements of the cavity resonance frequency. In the helical phase, a hybridized state of a higher-order helimagnon mode and a cavity mode — a helimagnon polariton—was found. Our results reveal a class of magnetic systems where strong coupling of microwave photons to nontrivial spin textures can be observed.
\end{abstract}

DOI: 10.1103/PhysRevB.99.140401

Introduction. Strong coupling between microwave photons and particle ensembles is a general phenomenon in light-matter interactions that has been observed in a broad range of condensed-matter systems, including ensembles of magnetically ordered spins [1-6], paramagnetic spins [7-10], and two-dimensional electron systems [11-13]. A common feature of ensemble coupling is that the coupling strength between a photon and $N$ particles scales with the square root of $N, g_{N}=g_{0} \sqrt{N}$, in accordance with the Dicke model [14-16]. Studies on strong coupling in spin systems are particularly interesting due to possible applications of hybrid spin-ensemble-photon systems for quantum information processing as quantum memories $[17,18]$ and quantum transducers [19]. The spin-ensemble coupling strength can be extremely large in magnetically ordered systems due to their high spin densities, and extensive studies of strong coupling to magnons - the quanta of spin-wave excitations in magnetically ordered systems — have been performed recently in experiments on ferrimagnetic insulators. In particular, new magnon-cavity-coupling phenomena have been observed in yttrium iron garnet, such as coherent coupling between a magnon and a superconducting qubit [20], microwave-tooptic-light conversion [21,22], cavity-mediated coherent coupling between multiple ferromagnets [23,24], spin pumping in a coupled magnon-photon system [25], and other phenomena [26-30].

\footnotetext{
*Present address: NTT Basic Research Laboratories, NTT Corporation, Atsugi, Kanagawa 243-0198, Japan; leonid.abdurakhimov.nz@hco.ntt.co.jp

${ }^{\dagger}$ Present address: Berlin Joint EPR Lab, Fachbereich Physik, Freie Universität Berlin, D-14195 Berlin, Germany.

${ }^{\ddagger}$ h.kurebayashi@ucl.ac.uk
}

So far, most studies of strong coupling in magnetic materials have focused on ferrimagnetic materials with the $\left(\mathbf{S}_{i} \cdot \mathbf{S}_{j}\right)$ like Heisenberg exchange interaction between neighbor spins $\mathbf{S}_{i}$ and $\mathbf{S}_{j}$. In these materials, all spins are collinear in the ground state, and mainly the uniform precession ferromagnetic mode, or the Kittel mode, has been used in the studies of magnon-photon coupling in those systems. However, there is a growing interest in the coupling of photons to noncollinear and other nontrivial spin systems [31,32]. In chiral magnets, the spin-spin exchange interaction consists of two terms; besides the symmetric Heisenberg interaction which favors collinear spin structures, there is an additional antisymmetric $\left(\mathbf{S}_{i} \times \mathbf{S}_{j}\right)$-like Dzyaloshinskii-Moriya (DM) interaction which tends to twist neighbor spins. As a result of the interplay between Heisenberg and DM exchange interactions, various noncollinear spin textures can be formed in chiral magnets, such as helical, conical, and skyrmion spin structures [see Fig. 1(a)]. Studies of the coupling between microwave photons and nontrivial spin textures is a potentially rich and largely unexplored area.

The chiral magnetic insulator copper-oxoselenite $\mathrm{Cu}_{2} \mathrm{OSeO}_{3}$ crystallizes in a noncentrosymmetric cubic structure with 16 copper ions $\mathrm{Cu}^{2+}$ per unit cell (space group $P 2{ }_{1} 3$, lattice constant $a \approx 8.93 \AA$ [33]). The basic magnetic building block of $\mathrm{Cu}_{2} \mathrm{OSeO}_{3}$ is a tetrahedral cluster formed by four $\mathrm{Cu}^{2+}$ spins in a 3-up-1-down spin configuration, which behaves as a spin triplet with the total spin $S=1$ [34]. This magnetic structure has been visualized elsewhere [34,35]. Due to a combination of Heisenberg and DM exchange interactions, the system of $S=1$ clusters forms helical, conical, ferromagnetic, and skyrmion magnetic phases in an applied external magnetic field below the Curie temperature $T_{\mathrm{C}} \approx 60 \mathrm{~K}$, as shown in Fig. 1(b). Above $T_{\mathrm{C}}$, the system is 
(a)

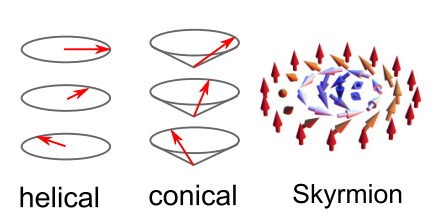

(c)

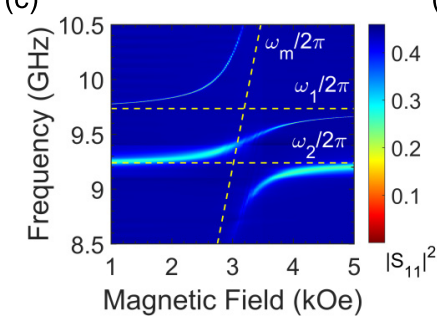

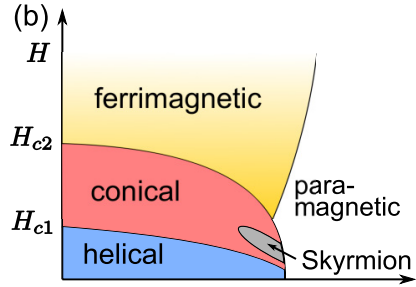

(d)

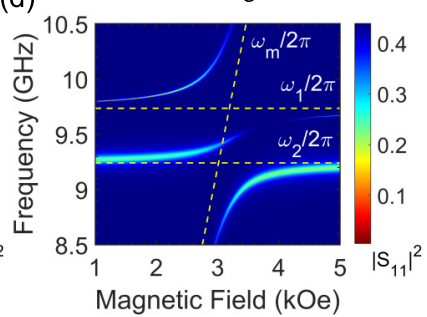

FIG. 1. (a) Schematic view of noncollinear spin textures. (b) Magnetic phase diagram of $\mathrm{Cu}_{2} \mathrm{OSeO}_{3}$. (c),(d) Strong coupling between ferrimagnetic mode of $\mathrm{Cu}_{2} \mathrm{OSeO}_{3}$ and multiple microwave cavity modes: (c) experimental data of microwave reflection $\left|S_{11}\right|^{2}$ as a function of the applied external field and the microwave probe frequency (temperature $T \approx 5 \mathrm{~K}$, microwave input power $P \approx$ $3 \mathrm{~mW}$ ), (d) microwave reflection $\left|S_{11}\right|^{2}$ calculated using Eq. (2) and parameters described in the text.

paramagnetic. Besides being a chiral magnet, $\mathrm{Cu}_{2} \mathrm{OSeO}_{3}$ is also of particular interest due to its multiferroic and magnetoelectric properties [35-39].

In this Rapid Communication, we report a study of magnon-photon coupling in a $\mathrm{Cu}_{2} \mathrm{OSeO}_{3}$ system. In the collinear ferrimagnetic phase, we observed a strong-coupling regime between a microwave cavity mode and a uniform Kittel magnon mode, and the temperature dependence of the coupling strength was found to follow that of the square root of the net magnetization. In the noncollinear conical phase, a dispersive coupling regime between helimagnons and microwave photons was observed, and we demonstrate that the magnetic phase diagram can be determined by the measurement of the frequency of a cavity mode. In the noncollinear helical phase, normal-mode splitting was detected between a higher-order helimagnon mode and a cavity mode.

Experimental details. We performed microwave $X$-band spectroscopy studies of $\mathrm{Cu}_{2} \mathrm{OSeO}_{3}$ in the temperature range 5-100 K using a helium-flow cryostat [40]. A sample of single-crystal $\mathrm{Cu}_{2} \mathrm{OSeO}_{3}$ was inserted into a commercial Bruker MD5 microwave cavity consisting of a sapphire dielectric ring resonator mounted inside a metallized plastic enclosure. The sample mass was $m \approx 60 \mathrm{mg}$, corresponding to a total effective spin number $N \approx 7 \times 10^{19}$. The shape of the sample was close to semiellipsoidal, with the lengths of semiaxes being $1.5,1.5$, and $2 \mathrm{~mm}$, and a flat plane being oriented along the long ellipsoid axis. The orientation of crystallographic axes of the sample relative to the cavity axis was chosen arbitrarily. The cavity supported two microwave modes [40,41]: the primary mode $\mathrm{TE}_{01 \delta}$ with the resonance frequency of about $\omega_{1} / 2 \pi \approx 9.74 \mathrm{GHz}$ and the hybrid mode $\mathrm{HE}_{11 \delta}$ with the resonance frequency of about $\omega_{2} / 2 \pi \approx 9.24 \mathrm{GHz}$. We tuned the quality factor $Q_{1}$ of the primary-mode resonance by adjusting the position of

a coupling loop antenna. In our measurements, we used a slightly undercoupled cavity with $Q_{1} \approx 5 \times 10^{3}$. The quality factor of the hybrid-mode resonance did not depend on the position of the coupling antenna, and was about $Q_{2} \approx 100$. In our experiments, the microwave reflection $S$ parameter $\left|S_{11}\right|^{2}$ was measured as a function of external magnetic field and microwave probe frequency.

Results. Figure 1(c) shows typical data from microwave reflection measurements at temperature $T \approx 5 \mathrm{~K}$, obtained from raw experimental data by background-correction processing [40]. The input microwave power was $P \approx 3 \mathrm{~mW}$. Two avoided crossings are visible at the degeneracy points where two cavity modes would otherwise intersect a magnon mode. The magnon mode corresponds to a uniform spin precession (Kittel mode) with frequency $\omega_{m} / 2 \pi=\gamma\left(H_{0}+\right.$ $\left.H_{\text {demag }}\right)$, where $H_{0}$ is the applied magnetic field, $H_{\text {demag }}$ is the demagnetizing field, and $\gamma \approx 28 \mathrm{GHz} / \mathrm{T}$ is the electron gyromagnetic ratio. Here, we assume that anisotropy fields are small and can be neglected.

The interaction between two cavity modes and a magnon mode can be described by the following Hamiltonian in the rotating-wave approximation (RWA):

$$
\begin{aligned}
\mathcal{H}_{0} / \hbar= & \omega_{1} a_{1}^{\dagger} a_{1}+\omega_{2} a_{2}^{\dagger} a_{2}+\omega_{m} m^{\dagger} m \\
& +g_{1}\left(a_{1}^{\dagger} m+a_{1} m^{\dagger}\right)+g_{2}\left(a_{2}^{\dagger} m+a_{2} m^{\dagger}\right),
\end{aligned}
$$

where $a_{1}^{\dagger}\left(a_{1}\right)$ is the creation (annihilation) operator for microwave photons at frequency $\omega_{1}, a_{2}^{\dagger}\left(a_{2}\right)$ is the creation (annihilation) operator for microwave photons at frequency $\omega_{2}, m^{\dagger}(m)$ is the creation (annihilation) operator for magnons at frequency $\omega_{m}$, and $g_{1}\left(g_{2}\right)$ is the coupling strength between the magnon mode and the first (second) cavity mode.

In order to extract numerical values of coupling strengths $g_{1}$ and $g_{2}$ and other parameters from the experimental data, we used the following equation obtained from input-output formalism theory [40]:

$$
\left|S_{11}\right|^{2}=\left|-1+\frac{\kappa_{1}^{(\mathrm{c})} F_{2}+\kappa_{2}^{(\mathrm{c})} F_{1}-2 \sqrt{\kappa_{1}^{(\mathrm{c})} \kappa_{2}^{(\mathrm{c})}} F_{3}}{F_{1} F_{2}-F_{3}^{2}}\right|^{2},
$$

where

$$
\begin{aligned}
& F_{1}=i\left(\omega_{1}-\omega\right)+\left(\kappa_{1}+\kappa_{1}^{(\mathrm{c})}\right) / 2+g_{1}^{2}\left(i\left(\omega_{m}-\omega\right)+\gamma_{m} / 2\right)^{-1}, \\
& F_{2}=i\left(\omega_{2}-\omega\right)+\left(\kappa_{2}+\kappa_{2}^{(\mathrm{c})}\right) / 2+g_{2}^{2}\left(i\left(\omega_{m}-\omega\right)+\gamma_{m} / 2\right)^{-1}, \\
& F_{3}=\sqrt{\kappa_{1}^{(\mathrm{c})} \kappa_{2}^{(\mathrm{c})}} / 2+g_{1} g_{2}\left[i\left(\omega_{m}-\omega\right)+\gamma_{m} / 2\right]^{-1},
\end{aligned}
$$

and $\kappa_{1}\left(\kappa_{2}\right)$ is the damping rate of the first (second) cavity mode, $\kappa_{1}^{(\mathrm{c})}\left(\kappa_{2}^{(\mathrm{c})}\right)$ is the coupling rate between the first (second) cavity mode and the output transmission line, and $\gamma_{m}$ is the damping rate of the magnonic mode. Damping rates represent linewidths (FWHM) of the corresponding modes.

We reproduce the data shown in Fig. 1(c) by using Eq. (2) with the following parameters: $g_{1} / 2 \pi \approx 600 \mathrm{MHz}, g_{2} / 2 \pi \approx$ $450 \mathrm{MHz}, \kappa_{1} / 2 \pi \approx 1 \mathrm{MHz}, \kappa_{1}^{(\mathrm{c})} / 2 \pi \approx 1 \mathrm{MHz}, \kappa_{2} / 2 \pi \approx$ $60 \mathrm{MHz}, \kappa_{2}^{(\mathrm{c})} / 2 \pi \approx 10 \mathrm{MHz}, H_{\mathrm{demag}} \approx 280 \mathrm{Oe}$, and $\gamma_{m} / 2 \pi \approx$ $50 \mathrm{MHz}$ [see Fig. 1(d)]. Thus, the coupling strengths are much greater than the damping rates of both cavity and magnon modes, $g_{1} \gg\left(\kappa_{1}+\kappa_{1}^{(\mathrm{c})}\right), \gamma_{m}$ and $g_{2} \gg\left(\kappa_{2}+\kappa_{2}^{(\mathrm{c})}\right), \gamma_{m}$, and 

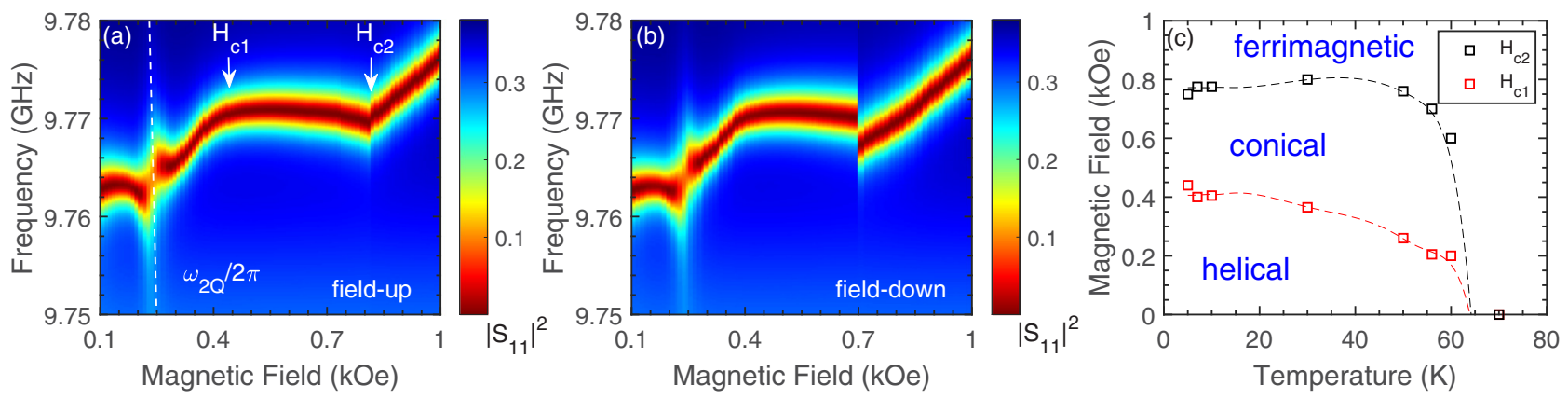

FIG. 2. Magnon-photon coupling in noncollinear magnetic phases. (a) Microwave reflection $\left|S_{11}\right|^{2}$ at 5 K. The magnetic-field sweep was performed from low to high field values ("field-up"). Dashed line corresponds to a higher-order helimagnon $k= \pm 2 Q$ mode, and an avoided crossing between the helimagnon mode and the cavity mode is clearly visible. $H_{c 1}$ and $H_{c 2}$ are critical magnetic fields of helical-to-conical and conical-to-ferrimagnetic phase transitions, respectively. (b) Microwave reflection $\left|S_{11}\right|^{2}$ at $5 \mathrm{~K}$. The magnetic-field sweep was performed from high to low values ("field-down"). The transition from ferrimagnetic to conical phase demonstrated hysteretic behavior. (c) The magnetic phase of the $\mathrm{Cu}_{2} \mathrm{OSeO}_{3}$ sample reconstructed from the field-up measurements of $H_{c 1}$ and $H_{c 2}$. The dashed lines are for eye guidance.

strong-coupling regimes are realized for both avoided crossings. From the obtained value of the ferrimagnetic resonance linewidth $\gamma_{m}$, we estimate the Gilbert damping parameter $\alpha \approx 2.6 \times 10^{-3}$ at $5 \mathrm{~K}$, which is consistent with the literature [42,43]. Cooperativity parameters are much higher than unity: $C_{1}=g_{1}^{2} /\left[\gamma_{m}\left(\kappa_{1}+\kappa_{1}^{(\mathrm{c})}\right)\right] \approx 3600$ and $C_{2}=$ $g_{2}^{2} /\left[\gamma_{m}\left(\kappa_{2}+\kappa_{2}^{(\mathrm{c})}\right)\right] \approx 60$. Moreover, ratios $g_{1} / \omega_{1} \approx 0.06$ and $g_{2} / \omega_{2} \approx 0.05$ are close to the condition of the ultrastrongcoupling regime $(g / \omega \geqslant 0.1)$, where the coupling strength is comparable with the frequency of the degeneracy point of an avoided crossing, and new physics beyond RWA can be explored $[3,4]$.

The obtained values of coupling strengths $g_{1}$ and $g_{2}$ between magnons and cavity modes are in relatively good agreement with the theoretical estimates

$$
g_{i}^{\text {(th) }}=\frac{\eta_{i}}{2} \gamma \sqrt{\frac{\mu_{0} \hbar \omega_{i}}{V_{i}}} \sqrt{2 S N} \quad(i=1,2),
$$

where $\mu_{0}$ is the vacuum permeability, $V_{1} \approx 230 \mathrm{~mm}^{3}\left(V_{2} \approx\right.$ $290 \mathrm{~mm}^{3}$ ) is the mode volume of the primary (hybrid) cavity resonance, and the coefficient $\eta_{1} \approx 0.85\left(\eta_{2} \approx 0.83\right)$ describes the spatial overlap between the primary (hybrid) cavity mode and the magnon mode [40]. Substituting other known parameters into the equations, we obtain $g_{1}^{\text {(th) }} / 2 \pi \approx 825 \mathrm{MHz}$ and $g_{2}^{\text {(th) }} / 2 \pi \approx 700 \mathrm{MHz}$. Slight discrepancies between theoretical and experimental values of coupling strengths can be caused by the excitation of additional $k \neq 0$ spin-wave modes in the sample which are visible as additional faint narrow lines in the experimental data, and the resulting reduction of the effective number of spins involved in the uniform Kittel-mode precession [44]. The difference in coupling strengths can be also related to the fact that the coupled system of magnons and photons is close to the ultrastrong-coupling regime mentioned above where the Hamiltonian (1) is not valid.

Coupling strengths $g_{1}$ and $g_{2}$ depended strongly on temperature [40]. In the ferrimagnetic phase, the effective number of spins is proportional to the net magnetization, and we found that the temperature dependence of the coupling rate could be fitted by square-root function of the net magnetization which is in good agreement with the results of studies of strong coupling in collinear ferrimagnetic systems [45]. In our exper- iments, anticrossing behavior was independent of microwave probe power, consistent with observations of strong coupling in other systems [1,5,7].

In order to study magnon-photon coupling in noncollinear spin textures, we performed measurements at low magnetic fields where the system exhibits helical and conical magnetic phases (see Figs. 2 and 3). We found that the frequency of a cavity resonance depended on the applied external magnetic field not only in the ferrimagnetic phase, but also in the helical and conical states [Figs. 2(a) and 2(b)]. We identify two features in microwave response at magnetic-field values $H_{c 1}$ and $H_{c 2}$ which can be attributed to helical and conical magnetic phase transitions in $\mathrm{Cu}_{2} \mathrm{OSeO}_{3}$, respectively [see Fig. 2(c)]. The observed transition from helical to conical phases is relatively smooth, which can be related to the fact that in the helical phase the spin system forms a multidomain structure of flat helices [35], and, since the external dc magnetic field was not aligned along the high-symmetry directions of the crystal structure in our measurements, domains gradually reoriented themselves with the increase of the magnetic field [46].

We suppose that the changes in the frequencies of cavity modes are caused by dispersive coupling between microwave photons and magnonic modes in the helical and conical phases-so-called helimagnons $[46,47]$. Frequencies of the fundamental $n= \pm 1$ helimagnon modes $\omega_{ \pm Q}$ lie well below the cavity resonance frequencies and depend weakly on the applied magnetic field. Since a full theoretical model of helimagnon-photon coupling would require detailed calculation of spectral weights of helimagnon modes [46], which is outside the scope of this paper, here we present a qualitative description of the dispersive magnon-photon coupling in $\mathrm{Cu}_{2} \mathrm{OSeO}_{3}$. In the helical phase $H<H_{c 1}$, the net magnetization $M$ is small [35], and helimagnon-photon coupling is very weak. In the conical phase $H_{c 1}<H<H_{c 2}$, the net magnetization is substantial, and the cavity mode is dispersively coupled to the fundamental helimagnon mode which results in the shift of the cavity resonance frequency. In the ferrimagnetic phase $H>H_{c 2}$, the net magnetization is close to its maximum value (saturation magnetization), the coupling strength to the Kittel mode is large, and the shift of the cavity resonance with the increase of the magnetic field is large. It should be noted that the magnetic-field 


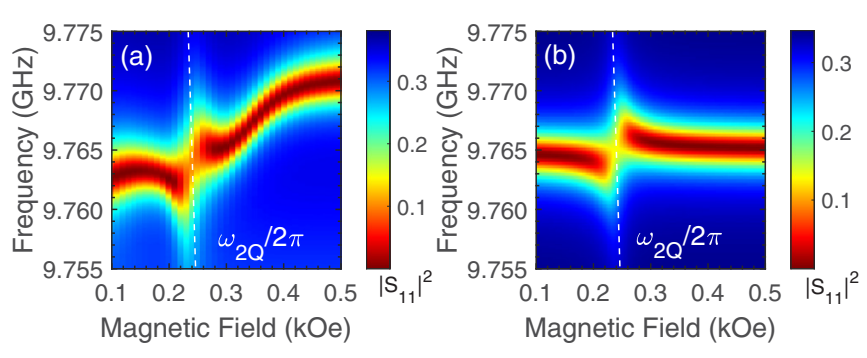

FIG. 3. Normal-mode splitting between a cavity mode and a higher-order helimagnon mode in the helical phase. (a) Microwave reflection $\left|S_{11}\right|^{2}$ at $5 \mathrm{~K}$. (b) Results of the numerical calculation of $\left|S_{11}\right|^{2}$ by using equations and parameters described in the text.

dependence of the cavity resonance frequency cannot be explained by the variation of a dc magnetic permeability of the material [40].

The value of the magnetic field $H_{c 2}$ of the conical-toferrimagnetic transition was found to be dependent on the direction of magnetic-field sweep [Figs. 2(a) and 2(b)]. This hysteretic behavior was observed only at low temperatures $T \lesssim 40 \mathrm{~K}$, and it can be related to either the competition between the conical state and the unusual "tilted conical" and skyrmion states recently reported in $[48,49]$ or the extension of the conical $n=1$ mode into the induced-ferrimagnetic phase (and vice versa) [47].

In the helical magnetic phase, we observed hybridization between a cavity mode and a higher-order helimagnon mode (see Fig. 3). By analogy with cavity magnon polaritons $[6,25,26,28]$, a hybrid helimagnon-photon state can be called a helimagnon polariton. In contrast to the ferrimagnetic mode, the dispersion curve of a helimagnon mode $\omega_{n Q}(H)$ exhibits a negative slope $\left(d \omega_{n Q} / d H<0\right)$. The helical phase is characterized by a multidomain structure of flat helices, where the propagation vectors of the different helices are pinned along the preferred axes of the system. The accurate description of helimagnons in the helical phase requires taking into account the cubic anisotropies of $\mathrm{Cu}_{2} \mathrm{OSeO}_{3}$ [46], which is outside the scope of this paper. Instead, we can use the analyitcal equation used for the description of magnons in the conical phase [47]:

$$
\omega_{n Q}=|n| \frac{\gamma B_{c 2}}{1+N_{d} \cdot \chi} \sqrt{n^{2}+(1+\chi)\left(1-\left(B_{0} / B_{c 2}\right)^{2}\right)}
$$

where $N_{d}$ is the demagnetization factor along the direction of the $Q$ vector, $\chi$ is the internal conical susceptibiliy $(\chi \approx 1.76$ for $\mathrm{Cu}_{2} \mathrm{OSeO}_{3}$ [46]), $B_{0}$ is the applied external field, and $B_{c 2}$ is the critical field for the transition between conical and ferrimagnetic phases ( $B_{c 2} \approx 0.08 \mathrm{~T}$ in our experiments). The mode number $n$ describes the relation between the helimagnon wave vector $k$ and the wave vector of the helical spiral $Q$, $k= \pm n Q$. By adjusting the parameters $n$ and $N_{d}$, we identify the observed helimagnon mode as an $n= \pm 2$ mode (the demagnetization factor $N_{d} \approx 0.1$ ), which is shown by the dashed line in Fig. 3.

In order to characterize the observed avoided crossing quantitatively, we use the following equation for the microwave reflection [3]:

$$
\left|S_{11}\right|^{2}=\left|-1+\frac{\kappa_{1}^{(\mathrm{c})}}{i\left(\omega_{1}-\omega\right)+\frac{\kappa_{1}+\kappa_{1}^{(\mathrm{c})}}{2}+\frac{g_{2 Q}^{2}}{i\left(\omega_{2 Q}-\omega\right)+\frac{\gamma_{2 Q}}{2}}}\right|^{2},
$$

where $\omega_{2 Q}$ and $\gamma_{2 Q}$ are the frequency and the linewidth of the helimagnon mode, respectively, and $g_{2 Q}$ is the coupling strength between the cavity mode and the helimagnon mode. We found that experimental data shown in Fig. 3(a) can be reproduced by using the following parameters: $g_{2 Q} / 2 \pi \approx$ $8.5 \mathrm{MHz}, \kappa_{1} / 2 \pi \approx 1.5 \mathrm{MHz}, \kappa_{1}^{(\mathrm{c})} / 2 \pi \approx 1.5 \mathrm{MHz}$, and $\gamma_{2 Q} / 2 \pi \approx 60 \mathrm{MHz}$ [see Fig. 3(b)]. Therefore, the observed normal-mode splitting can be attributed to the Purcell effect $\left(\kappa<g_{2 Q}<\gamma_{m}\right)$, where the decay of microwave cavity photons is enhanced due to their interaction with lossy magnons [3]. We could not detect the higher-order helimagnon mode $\omega_{2 Q}$ at temperatures $T \gtrsim 30 \mathrm{~K}$ which is consistent with the literature [47].

According to our numerical simulations, the mode overlapping between a cavity mode and higher-order $|n|=2$ helimagnon modes is suppressed as compared to the one for the Kittel ferromagnetic mode [40]. It should be noted that, according to the work [47], the direct interaction between a uniform microwave mode and $|n|=2$ helimagnons is negligible, but microwave photons can couple to $|n|=2$ helimagnons indirectly via fundamental $|n|=1$ helimagnons. Indeed, in the coordinate frame corotating with the spins around the helical-spiral wave vector $\mathbf{Q} \| \mathbf{z}$, the magnetic component of the $\mathrm{TE}_{01 \delta}$ cavity mode-which is spatially uniform within the sample volume in the laboratory frame of coordinates-corresponds to an effective microwave field with components $h_{1 x}^{\text {eff }} \propto \cos (Q z)$, and $h_{1 y}^{\text {eff }} \propto \sin (Q z)$. Therefore, the applied microwave magnetic field can excite directly only $|n|=1$ helimagnons which are characterized by the wave vector $|k|=Q$. However, in a cubic crystal, due to the fourth-order magnetic anisotropy $m_{x}^{4}+m_{y}^{4}+m_{z}^{4}$, where $\mathbf{m}$ is a unit vector in the direction of the magnetization, $n=\mp 1$ and $n= \pm 2$ helimagnon modes are hybridized [47]. Thus, the observed avoided crossing is caused by the double hybridization between microwave photons, $|n|=1$ helimagnons, and $|n|=2$ helimagnons.

We were not able to resolve coupling to magnetic excitations in the skyrmion phase in the measurements in the corresponding temperature range, presumably due to large detuning between the microwave cavity mode and skyrmion modes [50].

Conclusions. We performed a study of magnon-photon coupling in helical, conical, and ferrimagnetic phases of a chiral magnet $\mathrm{Cu}_{2} \mathrm{OSeO}_{3}$. We achieved a strong-coupling regime between a ferrimagnetic magnon mode and multiple microwave cavity modes. In the noncollinear conical phase, we observed the dispersive coupling between cavity modes and a fundamental helimagnon mode which allowed us to use a cavity mode as a probe for the sensing of magnetic phase transitions in $\mathrm{Cu}_{2} \mathrm{OSeO}_{3}$. In the noncollinear helical phase, we detected a normal-mode splitting between microwave photons and high-order helimagnons in the Purcell-effect regime. These findings establish an area of studies of strong-oupling phenomena in multiferroic chiral magnetic systems, paving 
the way for new hybrid systems consisting of nontrivial spin textures coupled to microwave photons via magnetic and magnetoelectric interactions.

Acknowledgments. This work was partly supported by the Grants-in-Aid for Scientific Research (Grants No. 18H03685, No. 17H05186, and No. 16K13842) from JSPS, and by the European Union's Horizon 2020 programme [Grant Agreements No. 688539 (MOS-QUITO) and No. 279781 (ASCENT)] as well as the Engineering and Physical Science Research Council UK through UNDEDD project (EP/K025945/1), and projects EP/K011987/1 and $\mathrm{EP} / \mathrm{S} 000798 / 1$.
[1] H. Huebl, C. W. Zollitsch, J. Lotze, F. Hocke, M. Greifenstein, A. Marx, R. Gross, and S. T. B. Goennenwein, Phys. Rev. Lett. 111, 127003 (2013).

[2] Y. Tabuchi, S. Ishino, T. Ishikawa, R. Yamazaki, K. Usami, and Y. Nakamura, Phys. Rev. Lett. 113, 083603 (2014).

[3] X. Zhang, C.-L. Zou, L. Jiang, and H. X. Tang, Phys. Rev. Lett. 113, 156401 (2014).

[4] M. Goryachev, W. G. Farr, D. L. Creedon, Y. Fan, M. Kostylev, and M. E. Tobar, Phys. Rev. Appl. 2, 054002 (2014).

[5] L. V. Abdurakhimov, Y. M. Bunkov, and D. Konstantinov, Phys. Rev. Lett. 114, 226402 (2015).

[6] M. Goryachev, S. Watt, J. Bourhill, M. Kostylev, and M. E. Tobar, Phys. Rev. B 97, 155129 (2018).

[7] I. Chiorescu, N. Groll, S. Bertaina, T. Mori, and S. Myiashita, Phys. Rev. B 82, 024413 (2010).

[8] D. I. Schuster, A. P. Sears, E. Ginossar, L. DiCarlo, L. Frunzio, J. J. L. Morton, H. Wu, G. A. D. Briggs, B. B. Buckley, D. D. Awschalom, and R. J. Schoelkopf, Phys. Rev. Lett. 105, 140501 (2010).

[9] Y. Kubo, F. R. Ong, P. Bertet, D. Vion, V. Jacques, D. Zheng, A. Dréau, J.-F. Roch, A. Auffeves, F. Jelezko, J. Wrachtrup, M. F. Barthe, P. Bergonzo, and D. Esteve, Phys. Rev. Lett. 105, 140502 (2010).

[10] J. D. Breeze, E. Salvadori, J. Sathian, N. M. Alford, and C. W. M. Kay, npj Quantum Inf. 3, 40 (2017).

[11] V. M. Muravev, I. V. Andreev, I. V. Kukushkin, S. Schmult, and W. Dietsche, Phys. Rev. B 83, 075309 (2011).

[12] G. Scalari, C. Maissen, D. Turčinková, D. Hagenmüller, S. De Liberato, C. Ciuti, C. Reichl, D. Schuh, W. Wegscheider, M. Beck, and J. Faist, Science 335, 1323 (2012).

[13] L. V. Abdurakhimov, R. Yamashiro, A. O. Badrutdinov, and D. Konstantinov, Phys. Rev. Lett. 117, 056803 (2016).

[14] G. S. Agarwal, Phys. Rev. Lett. 53, 1732 (1984).

[15] A. Imamoğlu, Phys. Rev. Lett. 102, 083602 (2009).

[16] B. M. Garraway, Philos. Trans. R. Soc., A 369, 1137 (2011).

[17] R. J. Schoelkopf and S. M. Girvin, Nature (London) 451, 664 (2008).

[18] G. Kurizki, P. Bertet, Y. Kubo, K. Mølmer, D. Petrosyan, P. Rabl, and J. Schmiedmayer, Proc. Natl. Acad. Sci. USA 112, 3866 (2015).

[19] S. Blum, C. O’Brien, N. Lauk, P. Bushev, M. Fleischhauer, and G. Morigi, Phys. Rev. A 91, 033834 (2015).

[20] Y. Tabuchi, S. Ishino, A. Noguchi, T. Ishikawa, R. Yamazaki, K. Usami, and Y. Nakamura, Science 349, 405 (2015).

[21] R. Hisatomi, A. Osada, Y. Tabuchi, T. Ishikawa, A. Noguchi, R. Yamazaki, K. Usami, and Y. Nakamura, Phys. Rev. B 93, 174427 (2016).

[22] J. A. Haigh, A. Nunnenkamp, A. J. Ramsay, and A. J. Ferguson, Phys. Rev. Lett. 117, 133602 (2016).

[23] X. Zhang, C.-L. Zou, N. Zhu, F. Marquardt, L. Jiang, and H. X. Tang, Nat. Commun. 6, 8914 (2015).
[24] N. J. Lambert, J. A. Haigh, S. Langenfeld, A. C. Doherty, and A. J. Ferguson, Phys. Rev. A 93, 021803(R) (2016).

[25] L. Bai, M. Harder, Y. P. Chen, X. Fan, J. Q. Xiao, and C.-M. Hu, Phys. Rev. Lett. 114, 227201 (2015).

[26] B. Yao, Y. S. Gui, J. W. Rao, S. Kaur, X. S. Chen, W. Lu, Y. Xiao, H. Guo, K.-P. Marzlin, and C.-M. Hu, Nat. Commun. 8, 1437 (2017).

[27] R. G. E. Morris, A. F. van Loo, S. Kosen, and A. D. Karenowska, Sci. Rep. 7, 11511 (2017).

[28] D. Zhang, X.-Q. Luo, Y.-P. Wang, T.-F. Li, and J. Q. You, Nat. Commun. 8, 1368 (2017).

[29] Y.-P. Wang, G.-Q. Zhang, D. Zhang, T.-F. Li, C.-M. Hu, and J. Q. You, Phys. Rev. Lett. 120, 057202 (2018).

[30] M. Harder, Y. Yang, B. M. Yao, C. H. Yu, J. W. Rao, Y. S. Gui, R. L. Stamps, and C.-M. Hu, Phys. Rev. Lett. 121, 137203 (2018).

[31] A. Osada, A. Gloppe, R. Hisatomi, A. Noguchi, R. Yamazaki, M. Nomura, Y. Nakamura, and K. Usami, Phys. Rev. Lett. 120, 133602 (2018).

[32] M. Mergenthaler, J. Liu, J. J. Le Roy, N. Ares, A. L. Thompson, L. Bogani, F. Luis, S. J. Blundell, T. Lancaster, A. Ardavan, G. A. D. Briggs, P. J. Leek, and E. A. Laird, Phys. Rev. Lett. 119, 147701 (2017).

[33] A. Larrañaga, J. L. Mesa, L. Lezama, J. L. Pizarro, M. I. Arriortua, and T. Rojo, Mater. Res. Bull. 44, 1 (2009).

[34] O. Janson, I. Rousochatzakis, A. A. Tsirlin, M. Belesi, A. A. Leonov, U. K. Rößler, J. van den Brink, and H. Rosner, Nat. Commun. 5, 5376 (2014).

[35] S. Seki, X. Z. Yu, S. Ishiwata, and Y. Tokura, Science 336, 198 (2012).

[36] S. Seki, S. Ishiwata, and Y. Tokura, Phys. Rev. B 86, 060403(R) (2012).

[37] Y. Okamura, F. Kagawa, M. Mochizuki, M. Kubota, S. Seki, S. Ishiwata, M. Kawasaki, Y. Onose, and Y. Tokura, Nat. Commun. 4, 2391 (2013).

[38] M. Mochizuki and S. Seki, J. Phys.: Condens. Matter 27, 503001 (2015).

[39] E. Ruff, P. Lunkenheimer, A. Loidl, H. Berger, and S. Krohns, Sci. Rep. 5, 15025 (2015).

[40] See Supplemental Materials at http://link.aps.org/supplemental/ 10.1103/PhysRevB.99.140401 for the experimental details, results of numerical calculations, and theoretical estimations.

[41] R. K. Mongia and P. Bhartia, Int. J. Microw. Millim.-Wave Comput.-Aided Eng. 4, 230 (1994).

[42] I. Stasinopoulos, S. Weichselbaumer, A. Bauer, J. Waizner, H. Berger, S. Maendl, M. Garst, C. Pfleiderer, and D. Grundler, Appl. Phys. Lett. 111, 032408 (2017).

[43] The reported value of Gilbert parameter corresponds to the damping of the Kittel mode. This should be distinguished from the damping of sharp spin-wave resonances for which Gilbert parameter can reach values on the order of $10^{-4}$ [42]. 
[44] I. Boventer, M. Pfirrmann, J. Krause, Y. Schön, M. Kläui, and M. Weides, Phys. Rev. B 97, 184420 (2018).

[45] H. Maier-Flaig, M. Harder, S. Klingler, Z. Qiu, E. Saitoh, M. Weiler, S. Geprägs, R. Gross, S. T. B. Goennenwein, and H. Huebl, Appl. Phys. Lett. 110, 132401 (2017).

[46] T. Schwarze, J. Waizner, M. Garst, A. Bauer, I. Stasinopoulos, H. Berger, C. Pfleiderer, and D. Grundler, Nat. Mater. 14, 478 (2015).

[47] M. Weiler, A. Aqeel, M. Mostovoy, A. Leonov, S. Geprägs, R. Gross, H. Huebl, T. T. M. Palstra, and S. T. B. Goennenwein, Phys. Rev. Lett. 119, 237204 (2017).
[48] F. Qian, L. J. Bannenberg, H. Wilhelm, G. Chaboussant, L. M. Debeer-Schmitt, M. P. Schmidt, A. Aqeel, T. T. M. Palstra, E. Brück, A. J. E. Lefering, C. Pappas, M. Mostovoy, and A. O. Leonov, Sci. Adv. 4, eaat7323 (2018).

[49] A. Chacon, L. Heinen, M. Halder, A. Bauer, W. Simeth, S. Mühlbauer, H. Berger, M. Garst, A. Rosch, and C. Pfleiderer, Nat. Phys. 14, 936 (2018).

[50] In the skyrmion phase, resonance frequencies of magnetic excitations are expected to be in the range $1-2 \mathrm{GHz}$ depending on the excitation type [51].

[51] Y. Onose, Y. Okamura, S. Seki, S. Ishiwata, and Y. Tokura, Phys. Rev. Lett. 109, 037603 (2012). 